\title{
(3) Acute Exacerbation
}

\author{
Hitoshi Nagano, M D
}

National Minami Fukuoka Chest Hospital, Fukuoka

The most important factor for deciding the prognosis of chronic respiratory failure is the acute exacerbation occurred in its chronic stable stage. The chronic respiratory failure may be defined as impairment of respiratory function to such degree that the arterial blood gas tensions depart from their normal values because of advancing diseases, and, in addition, as barely balanced conditions in spite of the abnormality of inner environment -back ground factorsof the body.

The acute exacerbation of chronic respiratory failure is a changed condition from this balanced state to which some additional cares should be taken. Patients whose respiratory failure was exacerbated become to have clinical disability such as breathlessness, and to feel the crisis of life.

The process of the changes from chronic respiratory failure to its acute exacerbation, and the management for it will be discussed from analysis of our own experiences.

Among patients admitted to our Hospital from 1970 to 1974,240 patients were diagnosed as acute exacerbation of chronic respiratory failure according to the present concept. Their arterial blood oxygen tentions were all below $70 \mathrm{mmHg}$. Almost all of the primary diseases during chronic stable state of respiratory failure were occupied by chronic respiratory diseases such as pulmonary tuberculosis, bronchial asthma, pulmonary emphysema, pulmonary cancer, and chronic bronchitis. Sixty seven cases of them were fatal (the mortality rate, $27.9 \%$ ).

The causes of disordering the balanced condition of inner environment of the patients were as follows: deterioration of pre- existing diseases $(9.6 \%)$, asthmatic attack $(25.4 \%)$, infectious complication of respiratory system $(42.4 \%)$, heart failure $(14.2 \%)$ and spontaneous pneumothorax (16.3\%). Complications of cerebrospinal diseases and disorders of digestive organs were also seen.

From asking patients' history in details, we could find some initiating stimulae - "triggers" - which might induce these complications or lead to break down chronic stable states of respiratory failure. Those were as follows; psychological factors such as post-childbirth, post-resection of uterus myoma, and pre-menstruation; changes of daily life such as sudden motion from rest, overwork, breathing cold air because of a cold wave sweeping in, and inevitable trip; iatrogenic factors such as long-term application of corticosteroids, digitalis, diuretics and medication of sedatives.

In addition to these triggers, we could detect premonitoring symptoms - "aurae"of complications and advancing of preexisting diseases which might show acute exacerbation beforehand. Those were as follows ; fatiguability, anorexia, weight loss, sleeplessness, and gastrointestinal symptoms.

These two important incidents could be secured by detailed observation and questionaire under co-operation of patient's family.

To catch the disorders of back ground factors, pathophysiological changes should be checked. Blood gas measurement is the most important thing. Lower $\mathrm{Pa}_{2}$ made prognosis worse, but some patients with $\mathrm{Pa}_{\mathrm{O}_{2}}$ above $60 \mathrm{mmHg}$ were fatal. Caution should not be directed just to anoxemia only. Respiratory acidosis was not always frequent, respiratory alkalosis existed also.

Jap J Med Vol 15, No 2 (April 1976) 
In $61 \%$ of the patients whose $\mathrm{Pa}_{\mathrm{O}_{2}}$ was below $40 \mathrm{mmHg}, \mathrm{PaCO}_{2}$ was above $45 \mathrm{mmHg}$. In accordance with advancing anoxemia, hypercapnia was increased. Therefore, although basic abnormality of arterial blood gas was hypoxemia, there are two types of hypoxemia: one with hypercapnia, another without hypercapnia.

As to clinical manifestations, cases of urine volume below $1000 \mathrm{ml}$ were $64 \%$ and those of below $500 \mathrm{ml}$ were $21 \%$. It was notworthy that cases whose sputum volume decreased to $5 \mathrm{ml}$ were $39 \%$, and that $60 \%$ of them were fatal. Those symptoms show deficit of water, which was accompanied with decreasing blood $\mathrm{Na}^{+}$and $\mathrm{Cl}^{-}$. Abnormality of the balance among water and salts also frequently seen. Polycythemia appeared in many cases. It is necessary to differentiate the polycythemia in respiratory failure either from haemoconcentration due to deficit of water or from secondary reaction to long lasting hypoxemia. Hyperproteinemia is likely to occur in the case of deficit of water, but hypoproteinemia, especially hy poalbuminemia is frequntly observable in the acute exacerbation. Low albumin/ globulin ratio, especially associated with an increase of blood globulin leads to high mortality, and thus problems between hypoxemia and inmmune mechanism would be offered to be studied. Besides, albumin itself acts as an anion which affected to the acid-base balance, and has a role of lowering the osmotic pressure, resulting the acceleration of edema formation.

It is suggested that when chronic respiratory failure was exacerbated, observation should be made out not only on pre-existing diseases and changes of arterial blood gas values, but on general conditions even on slight shifts of the various manifestation and physiologic findings from their values of chronic stable state.

Autopsy was done in 16 cases (male 13, and female 3 ) of 67 patients who died from acute exacerbation. All cases were over 50 years of age. Pre-existing diseases were pulmonary tuberculosis in 7 cases, pulmonary emphysema in 5, silicosis and fibrosis in 4 .
Dominant pathological reasons of deaths were complication of respiratory infection such as acute bronchitis and pneumonia, mucoid impaction, oxygen pneuminites, spontaneous pneumothorax and airway obstruction by food. Embolization of pulmonary artery and bleeding from digestive organ were also seen. Arterial blood gas values before death showed no certain inclination except those of severe hypercapnia as was seen in the cases with $\mathrm{CO}_{2}$ narcosis which was one of clinical reasons related to death. Degree of complication of right heart failure were detected by measuring the thickness of right ventricular wall, which were well correlated with the duration of inabilities for patients to move around during their stable states of chronic respiratory failure. In such cases with complication of right heart failure, their heart could not be compensated, so they would become to feel crisis of life. All 5 fatal cases with pulmonary emphysema had highly advanced brain edema and herniation of uncus gyri parahippocampalis. There would be relationship between these findings from autopsy an dramatic signs of breathlessness which are seen in patients with pulmonary emphysema.

\section{SUMMARY}

The acute exacerbation of respiratory failure would be defined as the changed condition from its chronic stable state to which some additional cares should be taken. Therefore, it should be diagnosed not only from arterial blood gas values, but also from abnormalities of back groud factors within the body and the stability of which has been constructed during its chronic shable state.

The primary diseases of chronic respiratory failure and causes of its acute exacerbation are stated. Findings from autopsy of cases died from exacerbation especially those of heart and brain are also discussed.

Thus, the clinical importance of management of acute exacerbation is, first, to try to avoid initiating stimulae, secondly, to detect premonitoring symptoms and begin 
adequate treatments as early as possible. Therefore, treatments are to correct abnormalities of back ground factors and to apply elaborated oxygen therapy in reference to arterial $\mathrm{CO}_{2}$ values with most suitable methods for each case. 